\title{
APPENDIX A: \\ FACULTY ROSTERS FROM THE \\ UNIVERSITY OF HEIDELBERG
}

The Faculty Roster as of 1560 :

\begin{tabular}{|c|c|c|c|}
\hline \multirow{4}{*}{$\begin{array}{l}\text { Theology } \\
\text { 1. Petrus } \\
\text { Boquinus } \\
\text { (Dekan) }\end{array}$} & Law & Medicine & Arts \\
\hline & \multirow{2}{*}{$\begin{array}{l}\text { Wendolinus } \\
\text { Heilmann }\end{array}$} & \multirow{3}{*}{$\begin{array}{l}\text { Jakob Curio } \\
\text { Petrus Lotichius } \\
\text { (Dekan) }\end{array}$} & Balch. Reisner \\
\hline & & & \\
\hline & Dionysius & & Georg Adam \\
\hline $\begin{array}{l}\text { 2. Paul Einhorn } \\
\text { (Unicornus) }\end{array}$ & Gravius (Graff) & Thomas Erastus & Wilhelm \\
\hline * & $\begin{array}{l}\text { Franciscus } \\
\text { Balduinus }\end{array}$ & & Xylander- \\
\hline & Caspar Agricola & & etc. \\
\hline & Nicolaus Cisner & & $\begin{array}{l}\text { Sigismund } \\
\text { Melanchthon }\end{array}$ \\
\hline
\end{tabular}

* Vacancy on the theological faculty due to the dismissal of Heshusius on Sept. 16, 1559.

** We know from other sources that Erastus occupied the second chair rather than the third chair.

UAH MS A. $160 / 7$ fol. 267. 
The Faculty Roster as of Dec. 22, 1568:

$\begin{array}{ll}\begin{array}{ll}\text { Theology } \\ \text { 1. Petrus }\end{array} & \text { Law } \\ \text { Boquinus-NT } & \begin{array}{c}\text { 1. Caspar } \\ \text { Agricola }\end{array} \\ \text { 2. Immanuel } & \text { 2. Berthold } \\ \text { Tremellius-OT } & \text { Redlich } \\ \text { 3. Girolamo } & \text { 3. Nicolaus } \\ \text { Zanchi- } & \text { Dobbin } \\ \text { Dogmatics } & \text { 4. Petrus } \\ & \text { Alostanus }\end{array}$

Medicine Arts

1. [Jakob Curio] ${ }^{\star}$ 1. Victorinus

2. Thomas

Strigel-Ethics

Erastus 2. Wilhelm

3. Sigismund Xylander-

Aristotle,

etc.

3. Hieronymus

Niger-Physics

4. Herman

Witekind-

Greek

5. Simon

Grynaeus-

Math.

6. Lambertus

Ludolphus

Pithopoeus

[Helm]-Latin

* Curio was not included on the list but must have still held the first chair.

UAH MS A. $160 / 9$ fol. 31. 
The Faculty Roster as of Dec., 1573:

$\begin{array}{cl}\text { Theology } & \text { Law } \\ \text { Petrus Boquinus- } & \text { Hugo Donellus } \\ \text { NT } & \text { Caspar Agricola } \\ \text { Immanuel } & \text { Nicolaus Dobbin } \\ \text { Tremellius-OT } & \\ \text { Girolamo } & \text { Petrus Alostanus } \\ \text { Zanchi- } & \\ \text { Dogmatics } & \end{array}$

Medicine Arts

Thomas Erastus Ulrich Faber

Albrecht Blaurer (Dekan)*

Ludwig Gravius (Graff)

Wilhelm

Xylander

Herman

Witekind

Simon Grynaeus

Matthaeus

Lannoius

[Lambertus

Ludolphus

Pithopoeus

$(\text { Helm) }]^{* *}$

* Faber's association with the faculty at this juncture is uncertain. See Drüll, ed., Heidelberger Gelehrtenlexikon 1386-1651, 1:147.

** Pithopoeus was not included on the list but was still a member of the arts faculty since he served as rector that year.

UAH MS A. $160 / 10$ fol. 62 . 
The Faculty Roster as of April 1577 with Remuneration:

\begin{tabular}{|c|c|c|c|}
\hline & & $\begin{array}{l}\text { Stipendia } \\
\text { Ordinaria }\end{array}$ & $\begin{array}{l}\text { Stipendia } \\
\text { extraordinaria }\end{array}$ \\
\hline Theology & $\begin{array}{l}\text { Petrus Boquinus } \\
\text { Immanuel Tremellius } \\
\text { Girolamo Zanchi }\end{array}$ & $\begin{array}{l}250 \mathrm{fl} . \\
200 \mathrm{fl} . \\
160 \mathrm{fl} .\end{array}$ & $120 \mathrm{fl}$. \\
\hline Law & $\begin{array}{l}\text { Hugo Donellus } \\
\text { Caspar Agricola } \\
\text { Nicolaus Dobbin }\end{array}$ & $\begin{array}{l}200 \mathrm{fl} . \\
200 \mathrm{fl} . \\
200 \mathrm{fl} .\end{array}$ & $\begin{array}{l}200 \mathrm{fl} . \\
50 \mathrm{fl} . \\
50 \mathrm{fl} .\end{array}$ \\
\hline Medicine & $\begin{array}{l}\text { Thomas Erastus } \\
\text { Albert Blaurer } \\
\text { Ludwig Gravius (Graff), } \\
\text { Rector }\end{array}$ & $\begin{array}{l}180 \mathrm{fl} . \\
160 \mathrm{fl} . \\
140 \mathrm{fl}\end{array}$ & $120 \mathrm{fl}$. \\
\hline $\begin{array}{l}\text { Philosophy and } \\
\text { Languages }\end{array}$ & $\begin{array}{l}\text { Herman Witekind } \\
\text { Simon Grynaeus } \\
\text { Lambertus Ludolphus } \\
\text { Pithopoeus (Helm) } \\
\text { Matthaeus Lannoius } \\
\text { Johannes Jungnitz } \\
\text { Theophil Mader }\end{array}$ & $\begin{array}{l}120 \mathrm{fl} . \\
120 \mathrm{fl} . \\
120 \mathrm{fl} . \\
120 \mathrm{fl} . \\
120 \mathrm{fl} . \\
120 \mathrm{fl} .\end{array}$ & $\begin{array}{l}30 \mathrm{fl} . \\
30 \mathrm{fl} .\end{array}$ \\
\hline
\end{tabular}

UAH MS A. $160 / 11$ fol. 77. 